\title{
EXTERIOR E INTERIOR GUERRA: EL FUNDAMENTO DE LA ALEGORÍA EN CALDERÓN
}

\author{
Luis Galván \\ Departamento de Filología \\ Facultad de Filosofia y Letras \\ Edificio de Bibliotecas \\ Universidad de Navarra \\ 31080 Pamplona. Navarra. España \\ lrgalvan@unav.es
}

[Anuario calderoniano (ISSN: 1888-8046), 4, 2011, pp. 169-180]

Que el auto sacramental es un elemento con papeles diversos en los contextos de la literatura, la religión, la política y la economía es cosa bastante sabida ${ }^{1}$; pero, por mucho que lo sea, no se puede perder nunca de vista, porque ello sucede en una época en la cual todos esos sistemas se van diferenciando y haciendo cada vez más autónomos. Hacía falta todo el talento de un Calderón de la Barca para explorar y explotar plenamente las posibilidades y tensiones que suscitaba semejante coyuntura, como voy a procurar mostrar haciendo referencia a algunos aspectos del auto La serpiente de metal.

${ }^{1}$ Bataillon, 1964; Arellano, 2001, pp. 95-98 y 115-146. 
Este auto fue estrenado en 1676 junto con Los alimentos del hombre. Excepcionalmente, hay información sobre el conjunto de la fiesta sacramental de aquel año: no solamente memorias de apariencias y compañías, sino también loas, piezas breves, música y danzas. Miguel Zugasti presenta los datos en el estudio preliminar de su excelente edición de Los alimentos ${ }^{2}$, por lo que no tengo nada que añadir aquí.

La serpiente de metal trata, en cuanto al aspecto histórico, de las experiencias del pueblo de Israel en la travesía del desierto, de Egipto a la tierra de Canaán. Se presentan las columnas de luz y de humo, el maná, el decálogo otorgado en el Sinaí, la adoración del becerro de oro, el combate contra Amalec, y como punto crítico y culminante la murmuración del pueblo contra Dios y Moisés, castigada con una plaga de serpientes venenosas, hasta que Moisés hace una de bronce que los israelitas han de mirar para salvarse de morir de las mordeduras (las fuentes son principalmente Éxodo, 16 y 32-34; y Números, 21).

Pretendo centrarme en un nivel básico de la constitución semántica del auto: la alegoría y la tipología desarrolladas a partir de dicha historia. Otros muchos temas que el asunto plantea habrán de quedar intactos, por requerir un análisis más detallado de lo que es posible en el marco de este trabajo; sin embargo, no puedo menos de mencionar, aunque sea tan solo marginalmente, el tratamiento de la murmuración, donde se anudan cuestiones de religión, moral y política. Es el problema de la opinión pública y la libertad de palabra opuestas a la autoridad del gobernante y el acatamiento que este necesita, problema que el auto intenta solucionar en términos religiosos — subrayando el fundamento divino del poder, que incluye la estructura de delegación- y sobre todo morales — censurando la conducta murmuradora, que se radica en los vicios capitales ${ }^{3}$.

La alegoría y la tipología son dos fenómenos semánticos relacionados, pero esencialmente distintos, y hay que tener siempre presente su diferencia para percibir las tensiones que se pueden generar. Mientras que el concepto de alegoría está bien implantado en la Retó-

2 Zugasti, 2009, pp. 7-24. Ver además las memorias de apariencias en Escudero y Zafra, 2003, pp. 163-162. Un aspecto complementario es la relación de este auto con la pintura barroca, especialmente la iconografía del maná y de la serpiente de metal, como pone de relieve Dietz, 2000.

${ }^{3}$ Ver Galván, 2009b; Gelz, en preparación. 
rica, desafortunadamente el de tipología es menos conocido y demasiadas veces se relega a especialistas en exégesis bíblica o en géneros literarios como este de los autos sacramentales, aunque se han ocupado de él filólogos tan relevantes como Erich Auerbach y Tzvetan Todorov ${ }^{4}$. Baste recordar brevemente la caracterización que hace Auerbach: la tipología «establece entre dos hechos o dos personas una conexión en la que uno de ellos no se reduce a ser él mismo, sino que además equivale al otro, mientras que el otro incluye al uno y lo consuma" ${ }^{5}$ : aquél es tipo o figura; el otro, antitipo o cumplimiento. Lo específico de esta relación, frente a la alegoría, es que «nos las habemos con la historicidad real tanto de la cosa significante como de la cosa significada»; es decir, los dos elementos son entes reales concretos ${ }^{6}$. El ámbito más conocido de aplicación de la tipología es la relación entre el Antiguo y el Nuevo Testamento, como es sabido — «et in Vetere Novum lateat, et in Novo Vetus pateat» ${ }^{7}$ - pero también se habla de tipología con relación a la posterior historia de la salvación - historia de la Iglesia y del mundo- y al final de los tiempos; y su empleo literario se extiende a otras áreas 8 .

Esta diferencia ha de completarse con la de símbolo y signo que propone Niklas Luhmann'. El símbolo representa lo en sí irrepresentable: es un elemento accesible que hace presente algo inaccesible; el signo, en cambio, hace referencia a algo que está ausente, que es inactual, ya sea por necesidad (como en el caso del símbolo), ya por simple contingencia ( $\mathrm{y}$ esto, por tanto, podría hacerse presente). Paradójicamente, el símbolo funciona según el modo de la identidad, el signo según el modo de la diferencia entre significante y significa$\mathrm{do}^{10}$. La alegoría — considera Luhmann- es un puente entre esas dos formas semánticas: se refiere a lo invisible, como el símbolo, pero se hace cargo de la diferencia, como el signo. Extendiendo estas ideas a la tipología, se la podría caracterizar primero de manera inversa: se re-

${ }^{4}$ Ver también Fabiny, 1992 y 2009; Galván, 2009a y 2011; para los autos, Arellano, 2001, pp. 59-64.

${ }^{5}$ Auerbach, 1998, p. 99.

${ }^{6}$ Auerbach, 1998, p. 100. Ver también Todorov, 1972 y 1974-1975.

${ }^{7}$ Agustín de Hipona, Quaestiones in Heptateuchum, II.73 (PL 34, col. 623).

${ }^{8}$ Ver, por ejemplo, Auerbach, 2002, pp. 180-193; Fabiny, 1992.

${ }^{9}$ Luhmann, 1997, pp. 271-288.

${ }^{10}$ Ver también Gadamer, 2003, pp. 41-52. 
fiere a lo visible, porque tipo y antitipo son históricamente reales; y se funda, como se verá, sobre la identidad. En un nivel ulterior, el vínculo entre los dos elementos visibles - sean cualesquiera en cada caso- se refiere nuevamente a lo inaccesible, la omnipotencia o la providencia de Dios que dispone las cosas creadas ${ }^{11}$. Las consecuencias fenomenológicas de semejante estructura saldrán a relucir cuando se considere el asunto del auto La serpiente de metal con ayuda de estos conceptos.

La historia de los israelitas en el desierto fue una de las privilegiadas por la visión tipológica. En uno de los más tempranos textos de lo que luego se llamó Nuevo Testamento, san Pablo escribe:

No quiero que ignoréis, hermanos, que nuestros padres estuvieron todos bajo la nube, y todos atravesaron el mar [...], y todos comieron el mismo alimento espiritual, y todos bebieron la misma bebida espiritual; porque bebían de la roca espiritual que los seguía, y la roca era Cristo. Pero la mayoría de ellos no agradó a Dios, puesto que cayeron muertos en el desierto. Estas cosas sucedieron como en figura para nosotros para que no codiciemos lo malo como lo codiciaron ellos [...]; ni tentemos al Señor, como lo tentaron algunos de ellos, y perecieron víctimas de las serpientes; ni murmuréis, como algunos de ellos murmuraron, y perecieron a manos del exterminador. Todas estas cosas les sucedían como en figura; y fueron escritas para escarmiento nuestro, para quienes ha llegado la plenitud de los tiempos (I Corintios, 10,1-11)

Aquí se encuentra la identidad entre lo viejo y lo nuevo: «la roca era Cristo» ${ }^{12}$, y, específicamente, la idea de que lo anterior en el tiempo es «figura» de lo posterior, mientras que lo posterior, por su parte, está situado en un nivel preeminente, de plenitud o finalidad.

En el evangelio según san Juan aparece, más claramente, la idea de superación: «Éste es el pan que ha bajado del cielo, no como el que comieron los padres y murieron: quien come de este pan vivirá eternamente» (Juan, 6, 58). Lo nuevo es más o mejor que lo viejo, como se declara también programáticamente en el prólogo (Juan, 1, 17-18).

${ }^{11}$ Ver Tomás de Aquino, Summa Theologica I, q. 1, art. 10.

${ }^{12}$ La misma identificación en Gálatas, 4, 22-26: Agar y Sara «son» (eisin) los dos testamentos. Por lo demás, este último pasaje es un lugar clásico para la amalgama de tipología y alegoría (De Lubac, 1947, pp. 182-183). 
A la luz de estos versículos hay que entender otro pasaje que se refiere de manera directa al episodio de las serpientes: «Igual que Moisés levantó la serpiente en el desierto, así debe ser levantado el Hijo del Hombre, para que todo el que crea tenga vida eterna en él» (Juan, 3, 14-15). Es decir, la serpiente de bronce, como el maná, daba una vida solamente temporal, mientras que Jesucristo crucificado, como la Eucaristía, dan la vida eterna.

Otro aspecto que se pone de manifiesto en los textos citados es la diversidad de orientaciones que puede tomar la lectura e interpretación, de acuerdo con el conocido dístico "Littera gesta docet, quid credas allegoria, / Moralis quid agas, quo tendas anagogia» ${ }^{13}$. En la carta de san Pablo se pone en primer lugar la aplicación moral, y resulta una forma antitética y más o menos paradójica. Plantea una analogía entre las situaciones de los israelitas antiguos y los cristianos actuales, y lo que pone de relieve es que los comportamientos pueden ser comparables, pero no deben ser iguales. La "figura» es, aquí, una suerte de ejemplo vitando. Sin embargo, no se limita a esto, porque se da también una relación entre los medios de subsistencia en el desierto y los medios de salvación: el pan y la bebida, bebida que brota de una roca, que es Cristo $^{14}$. Esto es objeto de la fe, es decir, materia de la lectura «alegórica» (en el último sentido indicado). La paradoja es, por tanto: contraste en lo moral, y analogía que llega a la identidad en el objeto de la fe. Por su parte, los versículos de san Juan apuntan más directamente a la fe y por medio de esta a la vida eterna, es decir, a la «anagogía».

Ahora se puede analizar cuál es la complejidad semántica de este repertorio de materiales y, por tanto, la del auto de Calderón que los sintetiza. En primer lugar, el asunto que le da título, la plaga de las serpientes, es interpretada por los personajes de la siguiente manera:

MOISÉS

[...] conmovido

Dios de las lástimas vuestras, viendo que misericordia

13 Nicolás de Lyra, «Prologus...» (PL 113, col. 28D).

${ }^{14}$ Por extensión, también la comida del desierto (el maná) es Cristo: la Eucaristía, tema que san Pablo trata inmediatamente después (I Corintios, 10, 15 y 11, 23-29); la identidad del maná y la Eucaristía es uno de los Leitmotiv de la liturgia del Corpus Christi (ver Tomás de Aquino, «Officium»). 
le pedís, $[. .$.$] me manda$ que exalte a la vista de ellas en la misteriosa vara de los prodigios aquesta sierpe. A verla, pues, venid; veréis que el que llegue a verla de las fieras mordeduras de otras sierpes convalezca.

Afecto 1. $\quad$ Dígalo yo, que al mirarla conozco que mi soberbia en esta exterior herida la interior salud preserva, pues la sanidad del cuerpo pasa a que el alma la tenga.

(vv. 2113-2136) $)^{15}$

$\begin{array}{ll}\text { MoIsÉs } & \text { Convino que en el metal } \\ & \text { tenga sola la apariencia } \\ & \text { del pecado, pero no } \\ & \text { que haya tenido ni tenga } \\ & \text { ni pueda tenerle quien } \\ & \text { en este se representa } \\ & \text { hoy para cuando exaltado } \\ & \text { en más noble vara penda. [...] } \\ & \text { Para sanar las dolencias } \\ & \text { del que mordido del áspid } \\ & \text { al pecador se semeja, } \\ & \text { no siendo él el pecador, } \\ & \text { convendrá que lo parezca. }\end{array}$

(vv. 2169-2186)

Lo que tenemos aquí es una semántica que combina propiedades del símbolo y del signo, entendidos según lo dicho anteriormente. En primer lugar, hay una referencia de lo visible presente (la serpiente de metal) a lo visible ausente (la crucifixión). Se percibe aquí la paradoja de la alegoría: no funciona estrictamente en el modo de la identidad («se representa», dice el auto, v. 2174), y por ello mismo tiene que

15 Cito el auto por el texto de mi edición crítica, en preparación. 
subrayar la semejanza y su justificación: conviene que el representante sea áspid, conviene que sea de metal, porque esto corresponde a características del representado, que es hombre, pero no pecador (compárese con la identidad, despreocupada de semejanzas, en las palabras de san Pablo antes citadas: «la roca era Cristo»).

En este punto es dificil aplicar la diferencia accesible/inaccesible, por la ambigüedad que envuelve la noción de lo inaccesible: para los personajes del auto, la crucifixión es contingentemente inaccesible, porque está demasiado distante en el futuro; para los espectadores, también es contingentemente inaccesible por haber sucedido muchos siglos antes; sin embargo, hay una cierta accesibilidad al conocimiento histórico, mediado verbal e institucionalmente. El paso a lo invisible e inaccesible es más claro en un segundo nivel: la relación entre la sanación del cuerpo y la salvación del alma. Se trata de un tema que pertenece a lo moral y a lo anagógico, y que el auto interpreta preferentemente en términos de moralidad, fundándose en el esquema dentro/fuera ${ }^{16}$.

Este es un hilo conductor del auto, que hace falta mirar con algún detalle. A propósito del primer episodio de la acción, la batalla de los israelitas contra Amalec, el personaje de la Idolatría comenta a Belfegor (el demonio):

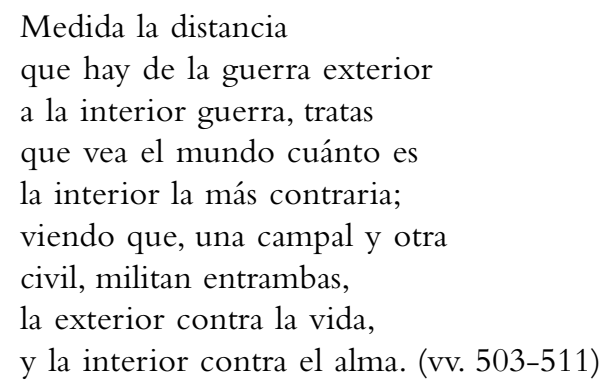

16 Ver Luhmann, 1987, pp. 114, 123-125. En relación con este auto, ver Poppenberg, 1998, pp. 204-206 (desarrollado en Poppenberg, 2009, pp. 333-336); es un caso particular del «drama del alma» con el motivo de la psicomaquia, que da forma pública y política al conflicto moral (Poppenberg, 2009, pp. 114-137). Ya Parker (1943, pp. 156-196) analizó un caso paradigmático de esta estructura, La cena del rey Baltasar. 
En el mismo sentido está tratado el "accidente» de parálisis que sufre María al murmurar de Moisés (vv. 1011-1036), y después le origina en lepra (vv. 1279-1283), y por último ocasiona la siguiente decisión:

MARÍA

\author{
Según mis ansias desean \\ ver a Moisés, he salido \\ del retiro que tenía, \\ oyendo que ya bajó \\ del monte; pues como yo \\ confiese la culpa mía, \\ a sus pies sanar espero. (vv. 1848-1854)
}

Por último, los ataques de las serpientes reciben de inmediato una interpretación moral: brotan del interior de los personajes y corresponden al carácter de cada uno. Reprocha Moisés a los hebreos, ante los «áspides, serpientes, víboras y culebras»:

Si aun perdonados no tienen
vuestros afectos enmienda,
no yo, vuestra poca fe,
idólatras, las engendra. (vv. 2007-2010)

En efecto, las serpientes atacan a cada "afecto» (pasión o vicio capital) ${ }^{17}$ de acuerdo con su modo de ser, más o menos convencional: «al respecto de la culpa / les corresponde la pena» (vv. 2069-2070). Muerden al soberbio y ambicioso en la frente; al avaricioso, en las manos; al lascivo, en los ojos; al iracundo y al envidioso, en el corazón; al goloso y perezoso, en la boca y las piernas. En alguna momento se formula la completa identificación entre serpiente y vicio: «¿Qué víbora había de ser / más que su lascivia mesma?» (vv. 2039-2040); y lo mismo vale para la sanación: "pues la sanidad del cuerpo / pasa a que el alma la tenga» (vv. 2135-2136).

Resulta clara entonces la diferencia entre esta forma de alegoría moral y la que se plantea en la carta de san Pablo a propósito de este mismo pasaje. En I Corintios, 10, se trata de una relación entre comportamientos. Lo que hicieron los israelitas y lo que pueden o deben

${ }^{17}$ Ver Zafra, 2007. 
hacer los fieles de Corinto son elementos de un mismo orden, exterior y accesible a la observación. Son comparables; por eso mismo se puede disuadir de la imitación y recomendar lo opuesto. En cambio, en el auto se produce el paso a otro orden, el interior de la persona, las causas y motivos de su comportamiento: afectos, pasiones o vicios. Lo exterior visible permite conocer lo interior.

Ahora bien, lo cierto es que no hay otro modo de acceso a la interioridad, pues se da la conocida paradoja de que esta no puede manifestarse como tal: ha de exteriorizarse ${ }^{18}$. Así, los episodios representados quedan en una situación ambigua: por un lado, necesitan estar respaldados por una interioridad, un fundamento, o no serían más que mera apariencia; por otro, son la garantía de que existe el interior fundamento que los respalda.

La situación ha de examinarse desde el punto de vista fenomenológico. Pues la estructura intencional de los actos de conciencia, tal como la describe Edmund Husserl, es análoga a la de la tipología: hay una expectación, o más en general una teleología, a la cual corresponde un cumplimiento. El «mero pensar» es insatisfactorio, desencadena un dinamismo que alcanza la satisfacción en la intuición de lo pensado. El cumplimiento o satisfacción son una vivencia de la identidad entre los objetos del primero y el segundo acto, pero es una identidad que incluye un incremento, una "preeminencia» del segun$\mathrm{do}^{19}$. Husserl señala las intenciones «significativas» como especialmente «vacías» y «necesitadas de plenitud»; en el otro extremo se encuentran actos especialmente caracterizados para funcionar como cumplimiento: la "plenitud de la representación", cuyo ideal solamente es alcanzado por la «percepción adecuada», con el «máximo de extensión, de

18 Es lo que Luhmann señala en su teoría del símbolo al decir que la diferencia accesible/inaccesible tiene una reentrada dentro de la cosa accesible que funciona simbólicamente (1997, p. 273).Ver también Luhmann, 1987, pp. 114-115, 123-124 y 201202; 1997, pp. 437-438 y 455. Este es también un tema favorito de la filosofía de Ortega, con la diferencia profundidad/superficie; ver Ortega 2005, pp. 34-42 y 2006, pp. 63-77. Dicha filosofia muestra que la deconstrucción de la «metafisica de la presencia» à la Derrida (1972) no son la única opción posible ante estas cuestiones.

${ }^{19}$ Ver en todo caso Husserl, 2006, vol. 2, pp. 621-627, 646 (investigación VI, cap. $1-3, \$ \$ 8,10,16)$. 
vivacidad y de realidad, justamente como aprehensión del pleno y total objeto mismo» ${ }^{20}$.

¿Qué forma de conciencia y de conocimiento se constituyen en el auto? La serpiente de metal, y no es pieza única en esto, ofrece una estructura intencional donde la percepción —el participar en los hechos los personajes, o el contemplar su representación los espectadores - no resulta suficiente, sino que necesita un cumplimiento. Lo que viene a cumplir ese acto primero no posee el carácter de la percepción, sino que tiene por objeto lo invisible: la vivencia moral interior, o directamente el ámbito de lo suprasensible y sobrenatural, la salvación. La fe es «fundamento" y "prueba», como se dice en la carta a los Hebreos $(11,1)$; pero aquí, paradójicamente, fundamento y prueba de lo que se $\mathrm{ve}^{21}$. La correspondencia de lo percibido, que es en sí insuficiente, con lo creído, que le da cumplimiento, es lo que proporciona la evidencia como vivencia de la verdad ${ }^{22}$.

En conclusión, retornando al marco social que se esbozó al principio, en una época de diferenciación y autonomización, el auto sacramental se presenta todavía como nudo y nexo de los sistemas religioso, político, moral y literario. Pone la posibilidad de conocer, de significar y comunicar en un fundamento divino. Claro está que la tradición del auto no había de ser bastante a contrarrestar la tendencia de la historia, y ni siquiera pudo sobrevivir a la autonomización de los órdenes mundanos (prohibición en el siglo XVIII). Además, la perspectiva postilustrada actual dificulta su comprensión, porque mira el auto como un fenómeno híbrido y extraño, mezcla de elementos pertenecientes a distintos sistemas, cuando en realidad manaba de la que fue fuente común a todos ellos.

${ }^{20}$ Husserl, 2006, vol. 2, pp. 653-659 (investigación VI, cap. 3, \ 23).

21 Para otra manifestación de esta estructura, en el juego de niveles de acción y representación, ver Galván, 2003.

${ }^{22}$ Ver Husserl, 2006, vol. 2, pp. 686 (investigación VI, cap. 5, \39). 


\section{BibLIOgRAFÍA}

Agustín de Hipona, Quaestionum in Heptateuchum libri septem, en Opera omnia, vol. 3, PL 34, Paris, Migne, 1845, col. 547-824.

Arellano, I., Estructuras dramáticas y alegóricas en los autos de Calderón, Kassel, Reichenberger, 2001.

Auerbach, E., Figura, Madrid, Trotta, 1998.

- Mímesis: la representación de la realidad en la literatura occidental, México, FCE, 2002, 9. ${ }^{\mathrm{a}} \mathrm{ed}$.

Bataillon, M., «Ensayo de explicación del auto sacramental», en Varia lección de clásicos españoles, Madrid, Gredos, 1964, pp. 183-205.

[Biblia:] Sagrada Biblia: Nuevo Testamento, Pamplona, Eunsa, 1999.

De Lubac, H., "“Typologie” et "Allégorisme”", Recherches de Science Religieuse, 34, 1947, pp. 180-226.

Derrida, J., La Voix et le phénomène, Paris, PUF, 1972.

Dietz, D. T., "La serpiente de metal de Pedro Calderón de la Barca», en El texto puesto en escena: estudios sobre la comedia en el siglo de oro en honor a Everett W. Hesse, ed. A. K. Stoll, B. Mujica, London, Tamesis, 2000, pp. 47-55.

Escudero, L., y Zafra, R., Memorias de apariencias y otros documentos sobre los autos de Calderón de la Barca, Kassel, Reichenberger, 2003.

Fabiny, T., The Lion and the Lamb: Figuralism and Fulfilment in the Bible, Art, and Literature, London, MacMillan, 1992.

- «Typology: Pros and Cons in Biblical Hermeneutics and Literary Criticism (From Leonhard Goppelt to Northrop Frye)», en Visiones para una poética: en el cincuentenario de "Anatomy of Criticism» de Northrop Frye, ed. L. Galván, número monográfico de Rilce: Revista de Filología Hispánica, 25.1, 2009, pp. 138-152.

Gadamer, H.-G., Die Aktualität des Schönen: Kunst als Spiel, Symbol und Fest, Stuttgart, Reclam, 2003.

Galván, L., "“Teatro de maravillas”: metamorfosis sobrenatural de los espacios en autos sacramentales de Calderón de la Barca", en Loca ficta: los espacios de la maravilla en la Edad Media y el Siglo de Oro, ed. I. Arellano, Madrid / Frankfurt, Iberoamericana / Vervuert, 2003, pp. 225-244.

- «Alegoría, tipología e intertextualidad: a propósito de Gonzalo de Berceo (Milagros de Nuestra Señora, XIX)", en Ars Bene Docendi: Homenaje al Profesor Kurt Spang, ed. I. Arellano,V. García Ruiz, C. Saralegui, Pamplona, Eunsa, 2009a, pp. 251-267.

— «Educación, propaganda, resistencia: Literatura y poder en teorías, tópicos y controversias de los siglos XVI y XVII», en Autoridad y poder en el Siglo de Oro, ed. I. Arellano, C. Strosetzki, E. Williamson, Madrid / Frankfurt, Iberoamericana / Vervuert, 2009b, pp. 51-87.

— "Meditación y semiología en Heráclito cristiano de Quevedo», en Eros di- 
vino: Poesía religiosa del Siglo XVII, ed. J. Olivares, Zaragoza, Universidad de Zaragoza, 2011 (en prensa).

Gelz, A., «El murmurador y la murmuración en la obra de Cervantes», ponencia presentada en el VII Congreso Internacional de la Asociación de Cervantistas (Universidad de Münster, 30-IX/4-X-2009), actas en preparación.

Husserl, E., Investigaciones lógicas, Madrid, Alianza, 2006, 2 vols.

Luhmann, N., Soziale Systeme: Grundriss einer allgemeinen Theorie, Frankfurt, Suhrkamp, 1987.

- Die Kunst der Gesellschaft, Frankfurt, Suhrkamp, 1997.

Nicolás de LyRA, «Prologus... de commendatione sacrae Scripturae in generali», en Walafridus Strabus, Glossa Ordinaria (Opera Omnia, I), PL 113, Paris, Migne, 1852, col. 25-30.

Ortega y Gasset, J., Meditaciones del Quijote, Madrid, Alianza, 2005.

- El hombre y la gente, Madrid, Alianza, 2006.

Parker, A. A., The Allegorical Drama of Calderon: An Introduction to the Autos Sacramentales, Oxford, Dolphin, 1943.

Poppenberg, G., "Quo tendas: hacia los autos poetológicos de Calderón», en Texto e imagen en Calderón: IX Coloquio Anglogermano sobre Calderón, ed. M. Tietz, Stuttgart, Steiner, 1998, pp. 202-207.

- Psique y alegoría: estudios del auto sacramental español desde sus comienzos hasta Calderón, Kassel, Reichenberger, 2009.

Todorov, T., «Introduction à la symbolique», Poétique, 3, 1972, pp. 273-308.

— «On Linguistic Symbolism», New Literary History, 6, 1974-1975, pp. 111134.

Tomás de Aquino, Summa theologica: Pars prima, seu Summa naturalis, ed. S.E. Fretté y P. Maré (Opera omnia, 1), Paris, L. Vivès, 1895.

- «Officium de Festo Corporis Christi», en Opera omnia, XXIX: Opuscula Theologica, Paris, L. Vivès, 1876, pp. 335-343.

Zafra, R., "El Baile de las Pasiones en los autos de Calderón», en El mundo maravilloso de los autos de Calderón, ed. I. Arellano y D. Reyre, Kassel, Reichenberger, 2007, pp. 229-239.

Zugasti, M., "Estudio preliminar», en P. Calderón de la Barca, Los alimentos del hombre, Kassel, Reichenberger, 2009, pp. 7-70. 